\title{
Die gemischte Episode bei bipolarer affektiver Störung - Änderungen im DSM-5 und aktuelle Behandlungsempfehlungen
}

\section{Mixed Episodes in Bipolar Disorders - Changes in DSM-5 and Treatment Recommendations}

Autoren

Institute
S. Köhler ${ }^{1}$, L. A. Stöver ${ }^{1,2}$, P. Sterzer ${ }^{1}$

Charité, Klinik für Psychiatrie und Psychotherapie, Campus Mitte

Psychiatrische Universitätsklinik der Charité im St. Hedwig-Krankenhaus, Berlin
Schlüsselwörter

- gemischte Episode

- DSM-5

- Behandlung

Key words

- mixed episode

- DSM-5

- treatment
Bibliografie

Dol http://dx.doi.org/

10.1055/s-0041-109017

Fortschr Neurol Psychiatr 2015;

83: 606-615 @ Georg Thieme

Verlag KG Stuttgart · New York .

ISSN 0720-4299

Korrespondenzadresse

PD Dr. med. Stephan Köhler

Charité, Klinik für Psychiatrie

und Psychotherapie,

Campus Mitte

Charitéplatz 1

10117 Berlin

stephan.koehler@charite.de

\section{Zusammenfassung}

$\nabla$

Die gemischte Episode stellt eine besondere Behandlungsbedingung im Rahmen affektiver Erkrankungen dar, die durch einen komplizierten und erschwerten Behandlungsverlauf (u.a. erhöhte Suizidrate) sowie ein schlechteres Therapieansprechen gekennzeichnet ist. Das DSM-5 hat als eine wesentliche Veränderung die Diagnose einer gemischten Episode abgeschafft und stattdessen sogenannte Episoden mit gemischten Merkmalen eingeführt. Dadurch sinkt die diagnostische Schwelle deutlich. Bezüglich der Pharmakotherapie gemischter Episoden ist die Datenlage insgesamt nicht ausreichend, jedoch zeigen sich Vorteile für die Behandlung mit atypischen Antipsychotika sowie Valproat und Carbamazepin.

\section{Einleitung}

Die gemischte Episode im Rahmen der bipolaren affektiven Störungen ist definiert über das gleichzeitige Auftreten depressiver und manischer Symptome. Im klinischen Alltag stellt dieses komplexe Erscheinungsbild der affektiven Störungen eine Herausforderung sowohl in Diagnostik als auch Behandlung dar. In der aktuellen Ausgabe des „Diagnostic and Statistical Manual of Mental Disorders“ (DSM-5) wurde eine wesentliche Veränderung in der Klassifikation der gemischten Episode hin zu einem dimensionalen Ansatz vorgenommen [1]. Dadurch sinkt die diagnostische Schwelle, was zu einer differenzierteren Wahrnehmung und Diagnostik führen kann und eine wichtige Ergänzung zu den ICD-10 Kriterien darstellt. Dennoch besteht weiterhin nur ein unzureichendes Wissen über eine spezifische Behandlung gemischter Episoden. Der vorliegende Artikel soll sowohl spezifische Merkmale und Besonderheiten in der Diagnostik nach DSM-5 bezüglich gemischter Episoden vor-

\section{Abstract \\ $\nabla$}

Mixed episodes in the course of affective disorders are challenging for any psychiatrist, because they are often characterized by a complicated and difficult treatment course (e.g. higher suicide rates) and a worse treatment outcome. In DSM-5, one of the main changes is that the diagnosis of a mixed episode was disestablished and replaced with affective episodes with so called "mixed features". This alteration markedly reduces the diagnostic threshold. The available literature on pharmacological treatment of mixed episodes is not sufficient; however, treatment with atypical antipsychotics, especially olanzapine and aripiprazole and the mood stabilizers valproate and carmabazepine has proved to be beneficial.

Auftreten manischer und depressiver Symptome im Rahmen des „manisch-depressiven Irreseins“ [2]. Gleichzeitig verwandte Kraepelin erstmals den Begriff der „Mischzustände“. Er unterschied in die Subtypen ängstliche (oder depressive) Manie, erregte (agitierte) Depression, gedankenarme Manie, manischer Stupor, ideenflüchtige Depression und gehemmte Manie. Wilhelm Weygandt setzte die Systematisierung des Konzepts der Mischzustände fort und stellte bereits 1899 fest: „Im manischen Anfall kann plötzlich die gehobene Stimmung in eine tief deprimierte übergehen, während im übrigen die flotteste Tobsucht wei- 
terbesteht mit ihrem Bewegungs- und Thatendrang, ihrer Ablenkbarkeit und Erregbarkeit, ihrem Rededrang und ihrer Ideenflucht [3].“

Kraepelin und Weygandt betonten bereits damals sowohl den protrahierten Verlauf als auch das Auftreten psychotischer Symptome im Rahmen von Mischzuständen. Als ein Erklärungsmodell für die Mischzustände sah Kraepelin die Veränderung von Stimmung, Kognition und Antrieb in jeweils unterschiedlicher Dynamik. Dieses Modell der Instabilität legt gleichzeitig die Mischzustände als eine eigenständige Subgruppe bipolarer affektiver Störungen nahe. In der Folge wurde manisch-depressiven Mischzuständen allerdings zunächst eine deutlich geringere Bedeutung beigemessen oder gar deren Existenz infrage gestellt [4]. Erst Ende der 70er Jahre wurde ausgehend von verschiedenen US-amerikanischen Arbeitsgruppen der Fokus erneut auf die manisch-depressiven Mischzustände gelegt (u.a. [5]), zunächst jedoch auf die Subtypisierung manischer und depressiver Syndrome (z. B. ängstliche Manie, agitierte Depression). Das Konzept der gemischten Episode als Kontinuum zwischen Depression und Manie wurde allerdings in den gängigen Klassifikationssystemen lange Zeit nicht umgesetzt (DSM III, DSM-III-R, DSM-IV, DSM-IV-TR, ICD-10). So wurde noch im DSM-IV-TR das gleichzeitige Zutreffen der Kriterien für eine manische und depressive Episode (im Rahmen einer Bipolaren Störung) für die Dauer von mindestens einer Woche gefordert, um die Diagnose einer gemischten Episode stellen zu können. Die ICD-10 hingegen fordert das gleichzeitige Auftreten zweier Symptomcluster, die während der meisten Zeit der Episode ( $\geq 2$ Wochen) im Vordergrund stehen.

Diese Kriterien führten zu einem sehr engen diagnostischen Fenster für gemischte Episoden bei gleichzeitig hoher Prävalenz subsyndromaler gemischter Zustandsbilder im klinischen Alltag. Zudem konnte basierend auf dem DSM-IV-TR eine gemischte Episode eigentlich nur für Bipolar-I-Störungen diagnostiziert werden, wohingegen dies für die Bipolar-II-Störung und die majore Depression nicht möglich ist. Insbesondere die Konstellation einer manischen oder depressiven Episode mit gleichzeitigem Auftreten weniger Symptome des jeweils entgegengesetzten Pols erlangte so keine diagnostische Bedeutung (u. a. $[6,7]$ ).

Insgesamt ist die Definition von Mischzuständen weiterhin Inhalt kontroverser Debatten. In einem Review zur bestehenden Literatur konnte jedoch festgestellt werden, dass das Konstrukt der Mischzustände unabhängig von den zugrunde liegenden diagnostischen Kriterien insgesamt sehr robust ist, leider auch bezüglich des komplexeren Krankheitsverlaufs, der höheren Rate an Komorbiditäten und des erhöhten Suizidrisikos [8].

\section{Prävalenz}

$\nabla$

Gemischte Episoden im Rahmen Bipolarer Störungen sind im klinischen Alltag häufig vorzufinden [9]. Aufgrund des engen diagnostischen Rahmens durch das DSM-IV und das ICD-10 variieren die Prävalenzraten (Punktprävalenz) in den Studien jedoch häufig, da teilweise Forschungskriterien angewendet wurden. Aktuelle Untersuchungen gehen von Prävalenzraten von 7 bis $28 \%$ unter den engen Kriterien der ICD-10 und des DSM-IV und bis zu 66\% unter eher weitgefassten Kriterien (z.B. drei Symptome des gegenüberliegenden Stimmungspols) aus [10]. Genauer untersuchten Vieta und Moralla (2010) basierend auf verschiedenen Kriterien die Prävalenzraten für gemischte Episoden: Dabei zeigte sich eine Prävalenz von 9\% (ICD-10), 13\% (DSM-IV-TR) bzw. 23\% (klinische Einschätzung der Behandler [11]).

\section{Suizidrisiko und Komorbiditäten}

\section{$\nabla$}

Insgesamt ist das Suizidrisiko bei Patienten mit einer gemischten Episode höher als bei Patienten mit „reinen“ Episoden. Sowohl die „gemischte Manie“ (Manie mit Symptomen einer Depression) als auch die „gemischte Depression“ (Depression mit Symptomen einer Manie) zeigen ein schlechteres Ansprechen auf die Behandlung und eine höhere Suizidrate gegenüber den „reinen“ Episoden [12]. In einer Untersuchung von 100 Patienten nach einem Suizidversuch lag die Prävalenz einer gemischten Depression (Kriterien ursprünglich nach Akiskal, übereinstimmend mit dem DSM-5, s. u.) bei $63 \%$. Insbesondere Reizbarkeit, Ablenkbarkeit und psychomotorische Erregung waren bei über $90 \%$ der Patienten mit einer gemischten Depression als Symptome festzustellen [12]. Gemischte Episoden sind gekennzeichnet durch ein insgesamt schlechteres Behandlungsergebnis und deutlich höhere Komorbiditätsraten (insbesondere Angststörungen, Substanzabhängigkeiten, Persönlichkeitsstörungen). Weiterhin sind gemischte Episoden wahrscheinlicher bei frühem Erkrankungsbeginn und es zeigt sich insgesamt eine höhere Frequenz an Episoden [8].

\section{Anmerkungen zum Konzept der gemischten Episode $\nabla$}

In der wissenschaftlichen Diskussion wird jedoch auch immer wieder angemerkt, dass trotz der Fülle von Beobachtungsstudien keine hinreichende Evidenz für eine eigene Diagnosekategorie der gemischten Episode vorliegt. Aus Sicht der Autoren ist die gemischte Episode als eigene Krankheitsentität nach den Kriterien in ICD-10 und DSM-IV zu unspezifisch. Vielmehr scheinen Mischzustände in vielen unterschiedlichen Formen klinisch apparent zu werden. Durch die Einführung der Specifier-Kriterien im DSM-5 wird versucht, das komplexe und stark heterogene Bild der Mischzustände und ihrer verschiedenen Zustandsbilder diagnostisch sensitiver und differenzierter zu erfassen. Dadurch ergibt sich eine stärkere diagnostische Nähe von Mischzuständen zu den beiden jeweiligen eindeutigen Ausprägungen bipolar-affektiver Störungen (Depression und Manie), die auch eine Ausrichtung differenzialtherapeutischer Überlegungen an diesen diagnostischen Kategorien nahelegt. Eine Bewertung des therapeutischen Nutzens dieses Ansatzes ist allerdings derzeit noch nicht möglich, da dafür noch keine hinreichende Evidenz aus prospektiven klinischen Studien vorliegt. Trotz der möglichen diagnostischen Nähe von Mischzuständen zu entweder manischen oder depressiven Episoden sollte beachtet werden, dass gemischte Episoden auch einige spezifische Merkmale zeigen, insbesondere hinsichtlich Verlauf und Therapie, weswegen sie besonderer Aufmerksamkeit bedürfen. Sowohl retrospektive als auch prospektive Studien ergaben gute Hinweise für die Stabilität gemischter Episoden, d.h. eine erhöhte Wahrscheinlichkeit einer gemischten Episode bei bereits stattgehabter gemischter Episode [13-15]. Auch bezüglich eines differenziellen Therapieansprechens, zum Beispiel im Vergleich zu rein manischen Episoden, liegt Evidenz für ein schlechteres Ansprechen von Patienten mit einer gemischten Episode auf die gängigen Therapiestrategien vor (u.a. [6, 16]). Zusätzlich weisen Patienten mit einer gemischten Episode ein höheres Suizidrisiko, mehr Komorbiditäten und häufigere Rezidive bzw. mehr Episoden auf [8] als Patienten mit reinen manischen oder depressiven Episoden. Zusammengefasst sind dies wichtige Aspekte, die gemischte Episoden als eine besondere Herausforderung in der Diagnostik und Therapie bipolarer Erkrankungen nahelegen. 


\section{Veränderungen im DSM-5}

$\nabla$

Das DSM-5 versucht im Unterschied zu seinen Vorgängern erstmals den Ansatz einer kategorialen Diagnostik mit einem dimensionalen Ansatz zu verbinden. Für die Problematik, dass es im DSM-IV-TR keine Möglichkeit gab, subsyndromale gemischte Episoden zu diagnostizieren, wurde in DSM-5 mit der Einführung der Zusatzbezeichnung „with mixed features“ („mit gemischten Merkmalen“) Abhilfe geschaffen (Überblick $\bullet$ Tab. 1).

Im DSM-5 wurde die gemischte Episode, wie sie bisher im DSMIV-TR definiert wurde, entfernt und Zusatzkriterien für beide Pole einer affektiven Erkrankung wurden eingeführt. So kann die Zusatzbezeichnung „gemischte Merkmale“ („mixed features specifier") beim Vorliegen von mindestens drei Symptomen des jeweils gegenüberliegenden Pols vergeben werden. Dadurch können im DSM-5 subsyndromale, nicht überlappende Symptome vom gegenüberliegenden affektiven Pol diagnostisch zugeordnet werden. Diese Zusatzdiagnose kann im Rahmen einer Bipolar-I-Störung, bei hypomanen Episoden bei Bipolar-I- und -II-Störungen, bei der majoren Depression bei Bipolar-I- und -II-Störungen, bei nicht näher bezeichneten Bipolaren Störungen sowie bei der unipolaren Depression (major depressive disorder, MDD) angewendet werden. Damit wird insbesondere der klinischen Erfahrung entsprochen, in der häufig Symptome des gegenüberliegenden affektiven Pols zwar vorlagen, jedoch diagnostisch bisher nicht näher zugeordnet werden konnten $[17,18]$. Im DSM-5 können zukünftig auch bei der unipolaren Depression die Zusatzkriterien „gemischte Merkmale“ diagnostiziert werden. Hintergrund ist, dass durch diverse Studien belegt werden konnte, dass ein großer Anteil der Patienten mit einer unipolaren Depression ebenfalls Symptome vom gegenüberliegenden affektiven Pol zeigt. Bis zu 40\% (u.a. Bridge Studie; [19]) der Patienten mit einer unipolaren Depression wiesen danach sogenannte Bipolar-Soft-Signs auf, also Merkmale, die auf eine bipolare Erkrankung hinweisen [18]. Durch die insgesamt niedrigere diagnostische Schwelle wird ein stärkerer Fokus auf das Erkrankungsbild der gemischten Episode gelegt, was aus Sicht der Autoren, insbesondere wegen der schwierigeren klinischen Verläufe, von großer Bedeutung ist.

In der Literatur wird häufig ein Spektrum der Mischzustände diskutiert mit den zwei Extremausprägungen der dysphorischen oder gereizten Manie und der Depression mit manischen Symptomen [8]. Natürlich kann der Grad der Ausprägung der Symptomatik der beiden Stimmungspole stark variieren mit weiteren Subtypen des klinischen Erscheinungsbildes [20]. Im Folgenden sollen jedoch spezifische Charakteristika der jeweiligen Mischformen dargestellt werden.

\section{Manische Symptome während einer depressiven Episode („Gemischte Depression“) \\ $\nabla$}

Im Rahmen depressiver Episoden sind relativ häufig auch manische Symptome vorzufinden, was als „gemischte Depression“ oder auch „agitierte Depression“ bezeichnet wird [21]. Je nach Diagnosekriterien und Studiendesign variiert die Prävalenz von 20 bis $70 \%$ (für Bipolare Störungen und für die MDD; [22]). Patienten mit einer gemischten Depression haben häufiger eine Bipolare Störung, ein früheres Ersterkrankungsalter, eine längere Behandlungsdauer mit schlechterem Therapieansprechen und schlechterem Behandlungsergebnis [23]. Die häufigsten manischen Symptome in einer depressiven Episode sind Stimmungsinstabilität, Ablenkbarkeit, ein beschleunigter formaler Gedankengang (Ideenflucht, Gedankenrasen, Gedankendrängen) sowie psychomotorische Erregung (u. a. [6]), wohingegen andere manische Symptome wie Hypersexualität, euphorische Stimmung und Größenideen eher selten sind ( $\bullet$ Tab.2). Die psychomotorische Unruhe und die Antriebssteigerung sind wie bei einer manischen Episode eher ungerichtet und wenig produktiv, dabei jedoch gleichzeitig mit erhöhter Suizidalität assoziiert [24]. Eine Schwierigkeit in der neuen Diagnostik nach DSM-5 ist der Ausschluss überlappender Kriterien für die Zusatzdi-

Tab. 1 Merkmale der DSM-5-Kriterien für affektive Episoden mit gemischten Merkmalen.

\begin{tabular}{|c|c|c|}
\hline $\begin{array}{l}\text { DSM-5-Klassifikation der } \\
\text { affektiven Episode }\end{array}$ & $\begin{array}{l}\text { DSM-5-Kriterien für eine Episode mit der Zusatzbezeichnung „gemischte Merkmale“ } \\
\text { (with mixed features) }\end{array}$ & Anwendbarkeit \\
\hline \multirow[t]{8}{*}{ Manie } & $\begin{array}{l}\text { Die Kriterien für eine manische Episode müssen erfüllt sein mit mindestens } 3 \text { weiteren } \\
\text { Symptomen: }\end{array}$ & \multirow[t]{8}{*}{$\begin{array}{l}\text { Manische Episoden im Rah- } \\
\text { men einer Bipolar-I-Störung }\end{array}$} \\
\hline & $\begin{array}{l}\text { 1. Dysphorie oder depressive Verstimmung (S: Traurigkeit, Gefühl der Leere; F: klagsam, } \\
\text { weinerlich) }\end{array}$ & \\
\hline & 2. Vermindertes Interesse oder Freude an (fast) allen Aktivitäten (SF) & \\
\hline & 3. Psychomotorische Verlangsamung (F) & \\
\hline & 4. Müdigkeit oder Energieverlust & \\
\hline & 5. Gefühl der Wertlosigkeit oder exzessive unangebrachte Schuldgefühle (nicht reine & \\
\hline & Selbstvorwürfe oder ausschließlich auf die Krankheit bezogene Schuldgefühle) & \\
\hline & 6. Wiederkehrende Suizidgedanken oder Gedanken an den Tod (nicht Angst vor dem Tod) & \\
\hline \multirow[t]{9}{*}{ Depression } & Die Kriterien für eine depressive Episode müssen erfüllt sein mit mindestens 3 weiteren & \multirow{9}{*}{$\begin{array}{l}\text { Majore Depressionen im } \\
\text { Rahmen einer Bipolar-I- oder } \\
\text {-II-Störung, bei nicht näher } \\
\text { bezeichneten Bipolaren Stö- } \\
\text { rungen und bei unipolaren } \\
\text { Depressionen }\end{array}$} \\
\hline & Symptomen: & \\
\hline & 1. Gehobene Stimmung & \\
\hline & 2. Übertriebenes Selbstwertgefühl oder Größenideen & \\
\hline & 3. Ungewöhnliche Geschwätzigkeit oder Rededrang & \\
\hline & 4. Ideenflucht oder subjektives Gedankenrasen & \\
\hline & 5. Erhöhtes Aktivitätsniveau (im sozialen, beruflichen, schulischen oder sexuellen Bereich) & \\
\hline & $\begin{array}{l}\text { 6. Vermehrte Beteiligung an Aktivitäten mit möglichen unangenehmen Konsequenzen } \\
\text { (z. B. Verschuldung, sexuell-übertragbare Krankheiten) }\end{array}$ & \\
\hline & 7. Vermindertes Schlafbedürfnis & \\
\hline \multirow[t]{2}{*}{ Hypomanie } & Die Kriterien für eine hypomane Episode sind erfüllt sowie mindestens 3 weitere & Hypomane Episoden bei \\
\hline & Symptome, diese gleichen den „gemischten Merkmalen“ bei einer Manie, s. o. & Bipolar-I- oder -II-Störungen \\
\hline
\end{tabular}




\begin{tabular}{|c|c|}
\hline Hauptsymptome der „gemischten“ Manie & Hauptsymptome der „gemischten“ Depression \\
\hline 1. Erhöhte Stimmungsinstabilität (v. a. Gereiztheit) & 1. Erhöhte Stimmungsinstabilität (v. a. Gereiztheit) \\
\hline 2. Psychomotorische Unruhe & $\begin{array}{l}\text { 2. Psychomotorische Unruhe, häufig ungerichtet und } \\
\text { wenig produktiv (v. a. Agitiertheit, Impulsivität und } \\
\text { vermehrter Rededrang) }\end{array}$ \\
\hline 3. Ablenkbarkeit & 3. Ablenkbarkeit \\
\hline 4. Extrem reduziertes Schlafbedürfnis & $\begin{array}{l}\text { 4. Beschleunigter formaler Gedankengang (Ideenflucht, } \\
\text { Gedankenrasen, Gedankendrängen) }\end{array}$ \\
\hline $\begin{array}{l}\text { 5. Geringeres Auftreten von Größenideen, Euphorie } \\
\text { und Antriebssteigerung (im Vergleich zur „reinen“ } \\
\text { Manie) mit eher „positiven“ Aktivitäten }\end{array}$ & $\begin{array}{l}\text { 5. Geringeres Auftreten von Hypersexualität, euphorischer } \\
\text { Stimmung und Größenideen (im Vergleich zur Manie) }\end{array}$ \\
\hline 6. Angstsymptome & \multirow{3}{*}{$\begin{array}{l}\text { 6. Höhere Suizidalität bei Vorliegen von psychomotorischer } \\
\text { Unruhe }\end{array}$} \\
\hline 7. Schuldgefühle & \\
\hline 8. Suizidgedanken & \\
\hline
\end{tabular}

Tab. 2 Übersicht Symptome der gemischten Manie und der gemischten Depression. agnose mit gemischten Merkmalen wie psychomotorische Erregung, Stimmungsinstabilität und Ablenkbarkeit (siehe Diskussion). Da diese jedoch wesentliche Merkmale der gemischten Depression sind, besteht in dieser Hinsicht die Möglichkeit falsch-negativer Befunde [22]. Bezüglich der Behandlung ist, wie weiter unten dargestellt, die Datenlage insbesondere für die gemischte Depression gering und widersprüchlich, vor allem was die Rolle der Antidepressiva betrifft [25]. Die Gabe von Antidepressiva bei gemischten Episoden sollte auch unter Betrachtung des Switch-Risikos von einer depressiven in eine manische Episode sowie einer möglichen Phasenakzeleration kritisch betrachtet werden [26].

\section{Depressive Symptomatik in einer manischen Episode (,gemischte Manie“) \\ $\nabla$}

Subsyndromale depressive Symptome während einer manischen Episode („gemischte Manie“) sind mit einem Auftreten von 25 $40 \%$ insgesamt sehr häufig $[27,28]$. Charakteristisch sind erhöhte Stimmungsinstabilität und Ablenkbarkeit sowie ein extrem reduziertes Schlafbedürfnis bei gleichzeitig geringerem Auftreten von Größenideen, Euphorie und Antriebssteigerung mit eher „positiven Aktivitäten“ im Vergleich zu „reinen“ Manien [29]. Gereizte Stimmung, Angstsymptome, Schuldgefühle sowie Suizidgedanken sind ebenfalls häufige Merkmale der gemischten Manie ( $\bullet$ Tab.2). Swann und Kollegen ziehen daher Parallelen zur agitierten Depression [30]. Hinsichtlich der Ausprägung der manischen Symptomatik sind die Befunde insgesamt stark heterogen, so dass sich keine eindeutige Aussage formulieren lässt [23]. Das Symptombild kann bei besonders schweren gemischten Manien (u.a. mit psychotischen Symptomen) an Komplexität deutlich zunehmen [22, 31], obwohl bei Patienten mit einer gereizten Manie psychotische Symptome wahrscheinlicher sind [32]. Zusammenfassend sind vor allem die psychomotorische Unruhe und die ausgeprägte emotionale Instabilität (Gereiztheit) wichtige Parameter in der Diagnostik der gemischten Manie. Bezüglich der Behandlung ist die Studienlage insgesamt besser als für die gemischte Depression mit Hinweisen für eine Wirksamkeit insbesondere von sowohl atypischen Antipsychotika [33] als auch Valproat und Carbamazepin (siehe unten).

\section{Vorteile und Nachteile in der neuen Diagnostik nach DSM-5}

Die neuen „Mixed-features-specifier“-Kriterien des DSM-5 ermöglichen die Diagnostik gemischter Anteile in einer Episode deutlich niedrigschwelliger. Dabei wird der ursprüngliche Ansatz von Kraepelin im Sinne eines Stimmungsspektrums untermauert [9, 34]. Dies ist aus Sicht der Autoren insbesondere wegen des schwierigen klinischen Verlaufs eine wichtige Veränderung. Mit der Möglichkeit, manische Symptome auch im Rahmen einer unipolaren Depression zu klassifizieren, wird ebenfalls eine Brücke zwischen der Bipolaren Störung und der unipolaren Depression geschlagen. Diese Beobachtung könnte im Langzeitverlauf von Patienten mit einer gemischten unipolaren Depression auf das Vorliegen einer Bipolaren Störung aufmerksam machen. Obwohl aus Sicht der Autoren die neuen Kriterien für eine gemischte Episode positiv zu bewerten sind, gibt es auch Schwierigkeiten, die daraus erwachsen: Aufgrund des Absenkens der diagnostischen Schwelle erscheint die Unschärfe zwischen verschiedenen Diagnosen eher verstärkt. Dies muss insbesondere angesichts der Möglichkeit von Fehldiagnosen und daraus resultierenden Behandlungsentscheidungen unbedingt beachtet werden [19]. Kritisch wird in der aktuellen wissenschaftlichen Diskussion insbesondere der Ausschluss der überlappenden Kriterien psychomotorische Unruhe und Ablenkbarkeit für die Zusatzbezeichnung „mixed features specifier“ diskutiert: Begründet wurde dieses vor allem mit dem hohen Überlappungsgrad beider Symptome im Rahmen verschiedener psychischer Erkrankungen. Gleichzeitig zeigen aktuelle Untersuchungen, dass gerade die genannten Symptome als Hauptmerkmale gemischter Episoden zu bewerten sind [21, 22]. Für den klinischen Alltag bedeutet dies, dass beim Vorliegen von psychomotorischer Unruhe und Ablenkbarkeit besonders auf Symptome des gegenüberliegenden Pols geachtet werden muss.

\section{Therapie \\ $\nabla$}

Die Behandlung gemischter Episoden stellt eine besondere Herausforderung im klinischen Alltag dar. Die Datenlage ist insgesamt mit nur sehr wenigen randomisierten kontrollierten Studien (randomized controlled trials, RCTs), die gezielt diese Fragestellung untersuchen, gering. Deshalb basieren viele Behandlungsempfehlungen auf Sekundäranalysen aus Studien, die verschiedene pharmakologische Behandlungsstrategien bei Patienten mit einer Bipolaren Störung untersuchten und in die Patienten mit einer gemischten Episode inkludiert waren. Die daraus resultierenden Bewertungen der Wirksamkeit spezifischer Substanzen müssen daher mit Vorsicht betrachtet werden. Weiterhin ist die Wirksamkeit aller untersuchten Substanzen insgesamt schlechter für Patienten mit einer gemischten Episode. Dennoch soll im Folgenden basierend auf den zur Verfügung stehenden Daten ein Überblick über 
die Wirksamkeit verschiedener Substanzen zur Behandlung gemischter Episoden gegeben werden. Wichtig ist dabei weiterhin, dass die diagnostischen Kriterien für eine gemischte Episode teilweise stark variieren, insgesamt natürlich aber das DSM-IV bzw. die ICD-10 und frühere Versionen Grundlage der Diagnostik waren und nur wenige Untersuchungen die neuen „Mixed-features-specifier"-Kriterien des DSM-5 verwendet haben. Eine Übersicht über alle RCTs zur Akutbehandlung gemischter Episoden, deren Charakteristika und wesentliche Limitationen ist in $\bullet$ Tab. 3 dargestellt. Die Empfehlungen für die Pharmakotherapie sind in $\bullet$ Tab. 4 zusammengefasst.

\section{Methode}

$\nabla$

Es wurde im November 2014 eine Medline-Suche mit den Begriffen „bipolar“, „mixed“ und „randomized“ durchgeführt. Insgesamt konnten bei 428 Ergebnissen 35 Studien eingeschlossen werden, wobei nur Daten aus randomisierten und kontrollierten Studien unter Einschluss von Post-hoc-Analysen und Metaanalysen berücksichtigt wurden. Die Angabe unkontrollierter Studienergebnisse erfolgt lediglich bei komplett fehlender Evidenz für Substanzen aus kontrollierten Untersuchungen.

\section{Klassische Mood-Stabilizer \\ $\boldsymbol{\nabla}$}

\section{Lithium}

Für den klassischen Mood-Stabilizer Lithium, der in der S3-Leitlinie zur Behandlung der Bipolaren Störung als einzige Substanz zur Phasenprophylaxe den höchsten Evidenzgrad A erhalten hat, ist die Datenlage zur Wirksamkeit bei gemischten Episoden insgesamt für eine abschließende Beurteilung der Wirksamkeit unzureichend. In älteren Untersuchungen zeigten sich Hinweise, dass bei Patienten in einer manischen Episode mit zusätzlich depressiver Symptomatik Lithium möglicherweise geringer wirksam ist als Valproat [35]. Auch in der Erhaltungsphase bei Patienten mit einer eher gereizten Manie im Rahmen einer Bipolar-IStörung (hier definiert als zusätzlich depressive Symptome zur Manie) zeigten sich Hinweise für eine schlechtere Wirksamkeit von Lithium im Vergleich zu „reinen“ affektiven Episoden. In einer weiteren Post-hoc-Analyse war Lithium gegenüber Placebo gleichwertig für Patienten mit einer dysphorischen Manie bezüglich des Auftretens einer neuen Episode unabhängig von der Polarität [36], jedoch wurde diese Untersuchung insbesondere wegen einer unrepräsentativen Patientenpopulation stark diskutiert.

\section{Valproat}

In der Studie von Bowden und Kollegen (2006) zeigte sich eine signifikante Abnahme manischer Symptome bei Patienten mit gemischter Episode im Vergleich zu Placebo [37]. In einer Untersuchung bei Patienten mit einer bipolaren Depression und zumindest einem manischen Symptom zeigte sich ebenfalls für die depressive Symptomatik eine signifikant stärkere Abnahme gegenüber Placebo, so dass für beide Pole eine Wirksamkeit von Valproat bei der gemischten Episode nachgewiesen werden konnte [38]. Hinsichtlich der rezidivprophylaktischen Wirkung von Valproat konnte allerdings in der Untersuchung von Bowden et al. [36] keine bessere Wirkung gegenüber Placebo bei Patienten mit einer dysphorischen Manie festgestellt werden.

\section{Carbamazepin}

Für Carbamazepin zeigten sich teilweise widersprüchliche Ergebnisse. In der Studie von Weisler et al. (2004) zeigte sich kein Unterschied in der Reduktion der manischen Symptomatik im Vergleich zu Placebo bei gleichzeitig signifikanter Reduktion depressiver Symptome [39]. In einer weiteren RCT von Weisler und Kollegen zeigte sich hingegen sowohl für manische als auch depressive Symptome bei Patienten mit gemischter Episode ein signifikanter Rückgang im Vergleich zu Placebo [40].

\section{Lamotrigin}

Für Lamotrigin liegen nur sehr wenige kontrollierte Untersuchungen hinsichtlich der Wirksamkeit bei gemischten Episoden vor. Einzig in einer offenen Studie von Calabrese und Kollegen wurden 11 Patienten mit Mischzuständen mit Lamotrigin als Add-on zu Lithium oder Valproat oder als Monotherapie behandelt. Lamotrigin als Mono- und als Kombinationstherapie reduzierte depressive und manische Symptome signifikant [41]. In der Studie von Carlson et al. [42] war Lamotrigin ähnlich wirksam wie die Kombination aus Lamotrigin und Aripiprazol in der Rückfallprophylaxe manischer und gemischter Episoden (gemeinsame Auswertung). In einer Registerdatenanalyse zeigten sich Lamotrigin und Lithium ähnlich wirksam bei der Behandlung von Mischzuständen [43]. In zwei unveröffentlichten Studien (SCAA2008 und SCAA2009) unterschied sich Lamotrigin hingegen nicht von Placebo in der Behandlung manischer und gemischter Episoden. Insgesamt ist die Datenlage zu gering, um eine Behandlungsempfehlung geben zu können [44].

\section{Antipsychotika \\ $\nabla$}

\section{Aripiprazol}

In einer Untersuchung von Sachs und Kollegen (2006) zeigte sich für Patienten mit einer gemischten Episode eine Überlegenheit von Aripiprazol gegenüber Placebo sowohl für depressive als auch für manische Symptome [45]. Weitere Untersuchungen konnten diesen Effekt bestätigen [7, 46].

\section{Asenapin}

In einer Studie mit manischen Patienten und Patienten mit einer gemischten Episode zeigte ebenfalls Asenapin eine Überlegenheit in der Reduktion manischer Symptome gegenüber Placebo und eine ähnliche Wirksamkeit im Vergleich zu Olanzapin [47]. Einschränkend muss erwähnt werden, dass bei einer der verwendeten statistischen Auswertungsmethoden Asenapin in der Subgruppe der Patienten mit einer gemischten Episode am primären Endpunkt (Reduktion in der YMRS-Scale) der Studie keinen Unterschied zu Placebo zeigte. Auch eine aktuelle gepoolte Post-hoc-Analyse zweier RCTs konnte diesen Effekt gegenüber Olanzapin nachweisen [48].

\section{Olanzapin}

Zur Wirksamkeit von Olanzapin bei gemischten Episoden sind gegenwärtig am meisten Aussagen möglich. 3 RCTs konnten die Wirksamkeit von Olanzapin auf die manischen Symptome einer gemischten Episode nachweisen (u. a. [47, 49]). In einer aktuellen Post-hoc-Analyse zeigten sich Hinweise auf die antimanische Wirksamkeit von Olanzapin bei gemischten Episoden auch unter Verwendung der „mixed-features-specifier“-Kriterien des DSM-5 [50]. Hinsichtlich der Verbesserung depressiver Symptome ist die Datenlage nicht so eindeutig. Insgesamt scheint nur eine Überle- 
Tab. 3 Übersicht Studien zur Akutbehandlung gemischter Episoden.

\begin{tabular}{|c|c|c|c|c|c|c|}
\hline Autor und Jahr & $\mathbf{n}$ & Studienpopulation & Substanzen & Studiendesign & Ergebnis & Limitationen \\
\hline $\begin{array}{l}\text { Swann } \\
\text { et al., } 1997\end{array}$ & 179 & $\begin{array}{l}\text { Manie mit und ohne } \\
\text { depressive Symptome } \\
\text { (SADS) }\end{array}$ & $\begin{array}{l}\text { Valproat, Lithium, } \\
\text { Placebo }\end{array}$ & $\begin{array}{l}\text { doppelblinde } \\
\text { RCT }\end{array}$ & $\begin{array}{l}\text { Depressive Symptome sprechen bes- } \\
\text { ser auf Valproat als auf Lithium an }\end{array}$ & $\begin{array}{l}\text { kurze Studiendauer von } \\
3 \text { Wochen }\end{array}$ \\
\hline $\begin{array}{l}\text { Tohen } \\
\text { et al., } 2002\end{array}$ & 344 & $\begin{array}{l}\text { Manie oder gemischte } \\
\text { Episode bei Bipolar-I- } \\
\text { Störung (DSM-IV) }\end{array}$ & $\begin{array}{l}\text { Olanzapin + Val- } \\
\text { proat/Lithium oder } \\
\text { Placebo + Valproat/ } \\
\text { Lithium bei nicht } \\
\text { ausreichender Wir- } \\
\text { kung durch Lithium } \\
\text { oder Valproat }\end{array}$ & $\begin{array}{l}\text { doppelblinde } \\
\text { RCT }\end{array}$ & $\begin{array}{l}\text { Valproat + Olanzapin ist der Kombi- } \\
\text { nation von Valproat + PLC überlegen } \\
\text { (YMRS, HAM-D); Lithium + Olanzapin } \\
\text { genauso wirksam wie Lithium + PLC } \\
\text { bei Pat. mit gemischter Episode }\end{array}$ & $\begin{array}{l}\text { Post-hoc-Analyse, ge- } \\
\text { mischte Diagnose- } \\
\text { gruppe }\end{array}$ \\
\hline $\begin{array}{l}\text { Baker } \\
\text { et al., } 2003\end{array}$ & 246 & $\begin{array}{l}\text { Manie oder gemischte } \\
\text { Episode bei Bipolar-I- } \\
\text { Störung (DSM-IV), }\end{array}$ & Olanzapin, Placebo & $\begin{array}{l}\text { Post-hoc-Ana- } \\
\text { lyse zweier dop- } \\
\text { pelblinder RCTs } \\
\text { (Tohen et al., } \\
\text { 1999, 2000) }\end{array}$ & $\begin{array}{l}\text { bei gemischter Episode signifikante } \\
\text { Verbesserung der HAM-D mit Olan- } \\
\text { zapin vs. PLC (-11,45 vs. }-6,83) \text {, } \\
\text { manische Symptome signifikant } \\
\text { reduziert (YMRS Olanzapin }-11,82 \\
\text { vs. PLC }-5,70)\end{array}$ & $\begin{array}{l}\text { Post-hoc-Analyse mit } \\
\text { geringer Teststärke } \\
\text { ( } n=33 \text { mit gemischter } \\
\text { Episode) }\end{array}$ \\
\hline $\begin{array}{l}\text { Baker } \\
\text { et al., } 2004\end{array}$ & 344 & $\begin{array}{l}\text { dysphorische Manie } \\
\text { (HAM-D > } 20 \text { Punkte) }\end{array}$ & $\begin{array}{l}\text { Olanzapin + Val- } \\
\text { proat/Lithium oder } \\
\text { Placebo + Valproat/ } \\
\text { Lithium bei nicht } \\
\text { ausreichender Wir- } \\
\text { kung durch Lithium } \\
\text { oder Valproat }\end{array}$ & $\begin{array}{l}\text { Post-hoc-Ana- } \\
\text { lyse der Tohen } \\
\text { et al. (2002) } \\
\text { Studie }\end{array}$ & $\begin{array}{l}\text { Kombination aus Olanzapin + Lithi- } \\
\text { um/Valproat führt zu signifikant } \\
\text { stärkerer Reduktion depressiver } \\
\text { Symptome als PLC + Lithium/Valproat }\end{array}$ & $\begin{array}{l}\text { Post-hoc-Analyse für } \\
\text { spezifische Subgruppe } \\
\text { der Patienten mit ge- } \\
\text { mischten Episoden }\end{array}$ \\
\hline $\begin{array}{l}\text { Weisler } \\
\text { et al., } 2004\end{array}$ & 204 & $\begin{array}{l}\text { Manie oder gemischte } \\
\text { Episode bei Bipolar-I- } \\
\text { Störung (DSM-IV) }\end{array}$ & $\begin{array}{l}\text { Carbamazepin, } \\
\text { Placebo }\end{array}$ & $\begin{array}{l}\text { doppelblinde } \\
\text { RCT }\end{array}$ & $\begin{array}{l}\text { für Subgruppe gemischte Episode } \\
\text { KEIN signifikanter Unterschied zwi- } \\
\text { schen Carbamazepin und PLC, hinge- } \\
\text { gen HAM-D signifikant verbessert } \\
\text { unter Carbamazepin vs. PLC }\end{array}$ & $\begin{array}{l}\text { hohe Studienabbruchra- } \\
\text { te }(>50 \%), 3 \text { Wochen } \\
\text { Dauer }\end{array}$ \\
\hline $\begin{array}{l}\text { Khanna } \\
\text { et al., } 2005\end{array}$ & 290 & $\begin{array}{l}\text { Manie oder gemischte } \\
\text { Episode bei Bipolar-I- } \\
\text { Störung (DSM-IV) }\end{array}$ & $\begin{array}{l}\text { Risperidon, } \\
\text { Placebo }\end{array}$ & $\begin{array}{l}\text { doppelblinde } \\
\text { RCT }\end{array}$ & $\begin{array}{l}\text { Response (mind. } 50 \% \text { ige Verbesse- } \\
\text { rung des YMRS) bei } 73 \% \text { unter Ris- } \\
\text { peridon vs. } 36 \% \text { unter Placebo, auch } \\
\text { MADRS signifikant verbessert unter } \\
\text { Risperidon, keine Unterschiede zw. } \\
\text { Gruppe der gemischten und mani- } \\
\text { schen Episode }\end{array}$ & $\begin{array}{l}\text { nur } 3 \text { Wochen Dauer, } \\
\text { gemischte Diagnose- } \\
\text { gruppe }\end{array}$ \\
\hline $\begin{array}{l}\text { Weisler } \\
\text { et al., } 2005\end{array}$ & 239 & $\begin{array}{l}\text { Manie oder gemischte } \\
\text { Episode bei Bipolar-I- } \\
\text { Störung (DSM-IV) }\end{array}$ & $\begin{array}{l}\text { Carbamazepin, } \\
\text { Placebo }\end{array}$ & $\begin{array}{l}\text { doppelblinde } \\
\text { RCT }\end{array}$ & $\begin{array}{l}\text { YMRS unter Carbamazepin signifi- } \\
\text { kant PLC überlegen, hingegen kein } \\
\text { Unterschied bei depressiver Sympto- } \\
\text { matik (HAM-D) }\end{array}$ & 3 Wochen Dauer \\
\hline $\begin{array}{l}\text { Bowden } \\
\text { et al., } 2006\end{array}$ & 377 & $\begin{array}{l}\text { Manie oder gemischte } \\
\text { Episode bei Bipolar-I- } \\
\text { Störung (DSM-IV) }\end{array}$ & Valproat, Placebo & $\begin{array}{l}\text { doppelblinde } \\
\text { RCT }\end{array}$ & $\begin{array}{l}\text { Response ( } 50 \text { \%ige Verbesserung } \\
\text { des MRS) unter Valproat } 48 \% \text { vs. } \\
34 \% \text { PLC }\end{array}$ & $\begin{array}{l}\text { gemeinsame Auswer- } \\
\text { tung, explorativ: Valpro- } \\
\text { at für gemischte und } \\
\text { manische Symptome } \\
\text { gleich wirksam, wenig } \\
\text { repräsentative Patien- } \\
\text { tenpopulation }\end{array}$ \\
\hline $\begin{array}{l}\text { Ghaemi } \\
\text { et al., } 2007\end{array}$ & 18 & $\begin{array}{l}\text { Depression bei Bipolarer } \\
\text { Störung (DSM-IV), } 73 \% \\
\text { mit mind. } 1 \text { manischen } \\
\text { Symptom }\end{array}$ & Valproat, Placebo & $\begin{array}{l}\text { doppelblinde } \\
\text { RCT }\end{array}$ & $\begin{array}{l}\text { Verbesserung der MADRS unter Val- } \\
\text { proat mit } 13,6 \text { Punkten vs. 1,4 Punk- } \\
\text { ten unter PLC }\end{array}$ & $\begin{array}{l}\text { gemischte Symptomatik } \\
\text { definiert mit } 1 \text { mani- } \\
\text { schem Symptom, } \\
\text { geringe Fallzahl }\end{array}$ \\
\hline $\begin{array}{l}\text { Suppes } \\
\text { et al., } 2008\end{array}$ & 519 & $\begin{array}{l}\text { Manie oder gemischte } \\
\text { Episode bei Bipolar-I- } \\
\text { Störung (DSM-IV) }\end{array}$ & $\begin{array}{l}\text { Aripiprazol, } \\
\text { Placebo }\end{array}$ & $\begin{array}{l}\text { Post-hoc-Ana- } \\
\text { lyse von } 2 \text { dop- } \\
\text { pelblinden RCTs } \\
\text { (Sachs et al., } \\
2006, \text { Keck } \\
\text { et al., 2003) }\end{array}$ & $\begin{array}{l}\text { signifikant reduzierte YMRS und } \\
\text { MADRS für gemischte und manische } \\
\text { Episode unter Aripiprazol vs. PLC }\end{array}$ & $\begin{array}{l}\text { kurzer Zeitraum der } \\
\text { Studien von } 3 \text { Wochen, } \\
\text { Post-hoc-Analyse zweier } \\
\text { RCTs mit gemischten } \\
\text { Diagnosegruppen }\end{array}$ \\
\hline $\begin{array}{l}\text { Vieta } \\
\text { et al., } 2008\end{array}$ & 253 & $\begin{array}{l}\text { Manie oder gemischte } \\
\text { Episode bei Bipolar-I- } \\
\text { Störung (DSM-IV), resis- } \\
\text { tent auf Lithium oder } \\
\text { Valproat }\end{array}$ & $\begin{array}{l}\text { Lithium/Valproat + } \\
\text { Aripiprazol oder } \\
\text { Lithium/Valproat + } \\
\text { Placebo bei nicht } \\
\text { ausreichender } \\
\text { Response auf } \\
\text { Lithium/Valproat } \\
\text { Monotherapie }\end{array}$ & $\begin{array}{l}\text { doppelblinde } \\
\text { RCT }\end{array}$ & $\begin{array}{l}\text { Kombination aus Valproat/Lithium + } \\
\text { Aripiprazol führt zu signifikant stär- } \\
\text { kerer Reduktion manischer Sympto- } \\
\text { me(YMRS) als Valproat/Lithium + PLC }\end{array}$ & $\begin{array}{l}\text { gemischte Diagnose- } \\
\text { gruppe, keine einzelne } \\
\text { Auswertung }\end{array}$ \\
\hline
\end{tabular}


Tab. 3 (Fortsetzung)

\begin{tabular}{|c|c|c|c|c|c|c|}
\hline Autor und Jahr & $\mathbf{n}$ & Studienpopulation & Substanzen & Studiendesign & Ergebnis & Limitationen \\
\hline $\begin{array}{l}\text { Houston } \\
\text { et al., } 2009\end{array}$ & 202 & $\begin{array}{l}\text { Gemischte Episode } \\
\text { (DSM-IV) }\end{array}$ & $\begin{array}{l}\text { Valproat + Olanza- } \\
\text { pin oder Valproat + } \\
\text { Placebo nach nicht } \\
\text { ausreichend wirk- } \\
\text { samer Valproat- } \\
\text { Monotherapie }\end{array}$ & $\begin{array}{l}\text { doppelblinde } \\
\text { RCT }\end{array}$ & $\begin{array}{l}\text { Kombination aus Valproat + Olanzapin } \\
\text { ist Valproat + PLC bei depressiven und } \\
\text { manischen Symptomen überlegen }\end{array}$ & \\
\hline $\begin{array}{l}\text { Stahl } \\
\text { et al., } 2010\end{array}$ & 179 & Dysphorische Manie & $\begin{array}{l}\text { Ziprasidon, } \\
\text { Placebo }\end{array}$ & $\begin{array}{l}\text { Post-hoc-Ana- } \\
\text { lyse zweier ge- } \\
\text { poolter RCTs } \\
\text { (Keck et al., } \\
\text { 2003, Potkin } \\
\text { et al., 2006) }\end{array}$ & $\begin{array}{l}\text { Ziprasidon signifikant besser wirk- } \\
\text { sam als PLC hinsichtlich manischer } \\
\text { und depressiver Symptome (MRS } \\
\text { und HAM-D) }\end{array}$ & $\begin{array}{l}\text { Post-hoc-Analyse zweier } \\
\text { RCTs mit gemischten Di- } \\
\text { agnosegruppen }\end{array}$ \\
\hline $\begin{array}{l}\text { Vieta } \\
\text { et al., } 2010\end{array}$ & 493 & $\begin{array}{l}\text { Manie oder gemischte } \\
\text { Episode bei Bipolar-I- } \\
\text { Störung (DSM-IV) }\end{array}$ & $\begin{array}{l}\text { Paliperidon, } \\
\text { Quetiapin, Placebo }\end{array}$ & $\begin{array}{l}\text { doppelblinde } \\
\text { RCT }\end{array}$ & $\begin{array}{l}\text { YMRS unter Paliperidon signifikant } \\
\text { reduziert vs. PLC, nach } 12 \text { Wochen } \\
\text { Paliperidon und Quetiapin gleich- } \\
\text { wertig, unter Paliperidon vs. Queti- } \\
\text { apin höheres „Switch-Risiko“ in eine } \\
\text { Depression ( } 13,9 \text { vs. } 7,5)\end{array}$ & $\begin{array}{l}\text { gemischte Diagnose- } \\
\text { gruppe }\end{array}$ \\
\hline $\begin{array}{l}\text { Azorin } \\
\text { et al., } 2013\end{array}$ & 295 & $\begin{array}{l}\text { gemischte Episode bei } \\
\text { Bipolar-I-Störung (DSM- } \\
\text { IV-TR) }\end{array}$ & $\begin{array}{l}\text { Asenapin, } \\
\text { Olanzapin, Placebo }\end{array}$ & $\begin{array}{l}\text { Post-hoc-Ana- } \\
\text { lyse zweier RCTs } \\
\text { (McIntyre et al.) }\end{array}$ & $\begin{array}{l}\text { Woche 3: sign. Verbesserung der } \\
\text { YMRS und MADRS unter Asenapin vs. } \\
\text { PLC und Olanzapin, Woche 12: wei- } \\
\text { tere Verbesserung der YMRS und } \\
\text { MADRS unter Asenapin, keine statis- } \\
\text { tische Überlegenheit zu Olanzapin }\end{array}$ & $\begin{array}{l}\text { Post-hoc-Analyse zweier } \\
\text { RCTs mit gemischten } \\
\text { Diagnosegruppen }\end{array}$ \\
\hline $\begin{array}{l}\text { Suppes } \\
\text { et al., } 2013\end{array}$ & 55 & $\begin{array}{l}\text { Bipolar-II-Störung, ggw. } \\
\text { gemischte hypomane } \\
\text { Symptome (DSM-IV-TR) }\end{array}$ & Quetiapin, Placebo & $\begin{array}{l}\text { doppelblinde } \\
\text { RCT }\end{array}$ & $\begin{array}{l}\text { Quetiapin zeigte sich PLC überlegen } \\
\text { hinsichtlich depressiver Symptome, } \\
\text { jedoch nicht bei hypomanen Symp- } \\
\text { tomen }\end{array}$ & $\begin{array}{l}\text { Geringe Fallzahl, Defi- } \\
\text { nition der gemischten } \\
\text { Episode }\end{array}$ \\
\hline $\begin{array}{l}\text { Tohen } \\
\text { et al., } 2014\end{array}$ & 447 & $\begin{array}{l}\text { Manie mit oder ohne ge- } \\
\text { mischte Merkmale bei Bi- } \\
\text { polar-I-Störung (DSM-5) }\end{array}$ & Olanzapin, Placebo & $\begin{array}{l}\text { Post-hoc-Ana- } \\
\text { lyse dreier dop- } \\
\text { pelblinder RCTs } \\
\text { (Tohen et al., } \\
\text { 1999, } 2000 \text {, } \\
\text { Katagiri et al., } \\
2012 \text { ) }\end{array}$ & $\begin{array}{l}\text { Unter Olanzapin vs. PLC signifikante } \\
\text { Reduktion der YMRS, größte Effizienz } \\
\text { bei Patienten mit gemischten Merk- } \\
\text { malen bei starker Ausprägung der } \\
\text { depressiven Symptomatik }\end{array}$ & $\begin{array}{l}\text { Post-hoc-Analyse zweier } \\
\text { RCTs mit gemischten } \\
\text { Diagnosegruppen }\end{array}$ \\
\hline $\begin{array}{l}\text { Tohen } \\
\text { et al., } 2014\end{array}$ & 1214 & $\begin{array}{l}\text { Depression mit gemisch- } \\
\text { ten Merkmalen bei Bipo- } \\
\text { lar-I-Störung (DSM-5) }\end{array}$ & Olanzapin, Placebo & $\begin{array}{l}\text { Post-hoc-Ana- } \\
\text { lyse zweier dop- } \\
\text { pelblinder RCTs } \\
\text { (Tohen et al., } \\
\text { 2000, Katagiri } \\
\text { et al., 2012) }\end{array}$ & $\begin{array}{l}\text { signifikante Verbesserung der } \\
\text { MADRS vs. PLC nach } 6 \text { Wochen }\end{array}$ & $\begin{array}{l}\text { Post-hoc-Analyse, DSM- } \\
\text { 5-Kriterien nicht exakt } \\
\text { ermittelt }\end{array}$ \\
\hline
\end{tabular}

MRS Mania Rating Scale; YMRS Young Mania Rating Scale; MADRS Montgomery-Asberg Depression Rating Scale; HAM-D Hamilton Depression Scale; DSM Diagnostic and Statistical Manual of Mental Disorders; SADS Schedule for Affective Disorders and Schizophrenia; RCT randomized controlled trial; CGI Clinical Global Impression; PLC Placebo.

genheit von Olanzapin gegenüber Placebo bei Patienten mit einer gereizten Manie oder sehr hohen depressiven Werten bei Studieneinschluss gegeben zu sein [51]. Eine aktuelle Analyse [52] konnte jedoch in der Gesamtauswertung einen positiven Effekt von Olanzapin auch auf die depressive Symptomatik feststellen. Auch für die Langzeitbehandlung sind aus Subgruppenanalysen positive Befunde im Sinne eines reduzierten Rückfallrisikos für die Behandlung mit Olanzapin zu verzeichnen [53].

\section{Paliperidon}

In einer RCT von Berwaerts et al. (2012) zeigte Paliperidon $(12 \mathrm{mg} / \mathrm{d})$ eine signifikant bessere Wirkung in der Behandlung manischer Symptome gegenüber Placebo für Patienten mit gemischter Episode. Kein Unterschied konnte hinsichtlich der depressiven Symptome festgestellt werden. In einer weiteren Untersuchung [54] zeigte sich Paliperidon ähnlich wirksam wie Quetiapin in der Reduktion manischer Symptome bei gemischten Episoden, aber auch hier schlechter in der Effektivität gegenüber depressiven Symptomen. In der Langzeitprophylaxe nach einer gemischten Episode zeigte sich Paliperidon in der Zeit bis zum Auftreten einer neuen Episode (unabhängig von der Polarität) Placebo signifikant überlegen [55].

\section{Quetiapin}

In der bereits zitierten Untersuchung von Vieta et al. zur Wirksamkeit von Quetiapin erfolgte keine Differenzierung zwischen manischen Patienten und Patienten mit einer gemischten Episode. In einer aktuellen Studie konnte bei Patienten mit einer Bipolar-II-Erkrankung und gemischten Symptomen kein Unterschied zwischen Placebo und Quetiapin festgestellt werden [56].

\section{Risperidon}

Die einzige vorliegende kontrollierte Untersuchung zeigte eine signifikant stärkere Abnahme manischer Symptome während einer Behandlung mit Risperidon gegenüber Placebo. Hinsichtlich der depressiven Symptomatik erfolgte keine Subgruppenanalyse zwischen manischen Patienten und Patienten mit einer gemischten Episode, bei gleichzeitiger signifikanter Reduktion der depressiven 


\begin{tabular}{|c|c|c|c|c|}
\hline $\begin{array}{l}\text { Medikamente und } \\
\text { deren Wirkung bei } \\
\text { gemischten Episoden }\end{array}$ & $\begin{array}{l}\text { Reduktion } \\
\text { manischer } \\
\text { Symptome }\end{array}$ & $\begin{array}{l}\text { Reduktion } \\
\text { depressiver } \\
\text { Symptome }\end{array}$ & $\begin{array}{l}\text { Reduziertes } \\
\text { Rückfallrisiko }\end{array}$ & $\begin{array}{l}\text { Vergleich zu anderen Antipsychotika, } \\
\text { Zusatzinformationen }\end{array}$ \\
\hline \multicolumn{5}{|l|}{ Antipsychotika } \\
\hline Aripiprazol & + & + & * & $\begin{array}{l}\text { Gute Wirksamkeit auf manische und depres- } \\
\text { sive Symptome bei gemischten Episoden }\end{array}$ \\
\hline Asenapin & + & * & * & $\begin{array}{l}\text { In Reduktion manischer Symptome ähnliche } \\
\text { Wirksamkeit wie Olanzapin [38] }\end{array}$ \\
\hline Olanzapin & + & + & + & $\begin{array}{l}\text { Beste Datenlage für Akut- und Langzeit- } \\
\text { behandlung }\end{array}$ \\
\hline Paliperidon & + & - & + & $\begin{array}{l}\text { Bessere Wirkung auf manische als auf } \\
\text { depressive Symptome, Hinweise auf pos. } \\
\text { Rückfallprophylaxe }\end{array}$ \\
\hline Quetiapin & * & * & * & $\begin{array}{l}\text { Keine ausreichende Datenlage zur } \\
\text { Bewertung }\end{array}$ \\
\hline Risperidon & + & * & * & $\begin{array}{l}\text { Hinweise auch für Wirkung auf depressive } \\
\text { Symptomatik [47] }\end{array}$ \\
\hline Ziprasidon & + & + & * & $\begin{array}{l}\text { Ziprasidon scheint wirksam auf manische und } \\
\text { depressive Symptome gemischter Episoden }\end{array}$ \\
\hline \multicolumn{5}{|c|}{ Klassische Mood-Stabilizer } \\
\hline Lithium & - & * & - & $\begin{array}{l}\text { Hinweise, dass Lithium bei Vorliegen depres- } \\
\text { siver Symptomatik Valproat unterlegen ist, } \\
\text { wohl kein reduziertes Rückfallrisiko }\end{array}$ \\
\hline Valproat & + & + & - & $\begin{array}{l}\text { Gute Wirksamkeit auf manische und depres- } \\
\text { sive Symptome bei fehlender Wirksamkeit in } \\
\text { der Rezidivprophylaxe }\end{array}$ \\
\hline Carbamazepin & * & + & $*$ & \\
\hline Lamotrigin & - & * & * & Kann ggw. nicht empfohlen werden \\
\hline \multicolumn{5}{|c|}{ Kombinationsbehandlung } \\
\hline Valproat + Olanzapin & + & + & $*$ & $\begin{array}{l}\text { signifikant wirksamer bei gemischter Episode } \\
\text { als Valproat-Monotherapie, auch signifikant } \\
\text { stärkere Abnahme der depressiven Sympto- } \\
\text { matik als Valproat-Monotherapie }\end{array}$ \\
\hline Lithium + Olanzapin & + & * & * & $\begin{array}{l}\text { Kombi-Behandlung ist der Lithium-Monothe- } \\
\text { rapie nicht überlegen }\end{array}$ \\
\hline $\begin{array}{l}\text { Lithium/Valproat + } \\
\text { Quetiapin }\end{array}$ & * & $\begin{array}{l}{ }^{*} \text { (Lithium) } /+ \\
\text { (Valproat) }\end{array}$ & + & \\
\hline $\begin{array}{l}\text { Lithium/Valproat + } \\
\text { Aripiprazol }\end{array}$ & + & + & * & $\begin{array}{l}\text { Eine Untersuchung ohne zusätzlichen Effekt } \\
\text { auf das Rückfallrisiko unter Aripiprazol-Aug- } \\
\text { mentation im Vergleich zur Valproat-/Lithi- } \\
\text { um-Monotherapie }\end{array}$ \\
\hline
\end{tabular}

Tab. 4 Übersicht pharmakologischer Behandlungsstrategien bei gemischten Episoden und deren Evidenz.

+ signifikant besser als Placebo, - nicht signifikant besser als Placebo, * unzureichende oder widersprüchliche Datenlage.

Symptomatik gegenüber Placebo für die gesamte Kohorte [57]. In einer aktuellen unkontrollierten Untersuchung konnte bei zusätzlicher Gabe von Risperidon zu einem Phasenprophylaktikum (Lithium, Valproat, Lamotrigin) ebenfalls eine Abnahme der manischen Symptomatik gezeigt werden, die signifikant höher war als die Abnahme der depressiven Symptomatik [58].

\section{Ziprasidon}

Ziprasidon zeigte in einer Untersuchung von Keck und Kollegen bei manischen Patienten und Patienten mit einer gemischten Episode eine deutliche Reduktion manischer Symptome, ohne dass jedoch spezifische Unterschiede bei der gemischten Episode gegenüber Placebo angegeben wurden [59]. In einer Post-hoc-Subgruppenanalyse für Patienten mit dysphorischer Manie war Ziprasidon Placebo bei der Reduktion sowohl manischer als auch depressiver Symptome überlegen $[60,61]$.

Zusammenfassend wurde die Wirksamkeit der atypischen Antipsychotika in einer aktuellen Metaanalyse untersucht [33]. Dabei zeigte sich für die Gruppe der atypischen Antipsychotika insgesamt ein signifikanter Vorteil gegenüber Placebo in der Reduktion manischer Symptome im Rahmen einer gemischten Episode.
Hinsichtlich der depressiven Symptomatik ist dies hingegen nicht eindeutig zu beurteilen (u.a. fehlende Angaben). Einschränkend muss erwähnt werden, dass zum einen das Design (Placebo-Vergleich) günstig für den Nachweis einer Wirksamkeit ist, jedoch die Effektstärken dennoch überwiegend klein waren (standardized mean difference $<0,5$ ). Ein weiterer Aspekt, der kritisch betrachtet werden sollte, ist, dass es keine großen, kontrollierten Vergleichsstudien zwischen atypischen Antipsychotika und klassischen Stimmungsstabilisierern gibt, aber ein größeres Interesse hinsichtlich der Vermarktung atypischer Antipsychotika besteht. Es gibt keine Studien, die eine Wirksamkeit von typischen Antipsychotika bei gemischten Episoden nachweisen [62]. Psychotische Symptome im Rahmen von gemischten Episoden können jedoch auf typische Antipsychotika ansprechen [63].

\section{Kombinationsbehandlungen:}

Für wenige Kombinationsbehandlungen konnte in kontrollierten Untersuchungen auch für die Subgruppe von Patienten mit einer gemischten Episode eine Wirksamkeit festgestellt werden. 
In der Akutbehandlung zeigte sich auch die Augmentation von Valproat mit Olanzapin signifikant wirksamer als die Monotherapie bei Patienten mit gemischter Episode, was für die Augmentation von Lithium nicht der Fall war [64]. Auch für die depressive Symptomatik zeigte sich in der Kombinationsbehandlung mit Olanzapin eine signifikant stärkere Abnahme gegenüber der Monotherapie [65]. Die Wirksamkeit der Kombination aus Olanzapin und Valproat wurde auch in einer der wenigen Studien, die gezielt gemischte Episoden untersuchten, sowohl für die depressive als auch für die manische Symptomatik nachgewiesen [66]. In der Erhaltungstherapie war die Kombinationsbehandlung mit Quetiapin zusätzlich zu Lithium oder Valproat bei Patienten mit gemischter Episode hinsichtlich des Rückfallrisikos Placebo überlegen [67]. Auch die Kombination von Aripiprazol mit Valproat oder Lithium scheint der alleinigen Monotherapie mit Valproat oder Lithium überlegen zu sein [68]. Hingegen konnte für Aripiprazol in der Kombination mit Lithium oder Valproat kein zusätzlicher Effekt bezogen auf die Verhinderung gemischter Episoden gezeigt werden [69].

\section{Take Home Message}

- Gemischte Episoden stellen eine besondere Herausforderung im klinischen Behandlungsalltag dar, da sie häufig sehr heterogen sind, ein schlechtes Ansprechen auf Medikamente zeigen und zugleich eine besondere Schwere der Symptomatik aufweisen.

- Im DSM-5 wurde die Diagnose einer gemischten Episode zugunsten sogenannter „specifier“ Kriterien aufgegeben, so dass bereits wenige Symptome vom gegenüberliegenden affektiven Pol zur Zusatzdiagnose „mit gemischten Merkmalen" ausreichen.

- Pharmakologisch zeigen insbesondere die atypischen Antipsychotika Olanzapin und Aripiprazol und auch Valproat eine Wirksamkeit in der Behandlung gemischter Episoden.

Interessenkonflikt: Die Autoren geben an, dass kein Interessenkonflikt besteht.

\section{Literatur}

1 American Psychiatric Association. Diagnostic and Statistical Manual of Mental Disorders. Arlington, VA: American Psychiatric Publishing; 2013

2 Kraepelin E. Psychiatrie. 7. Aufl. Leipzig: Barth; 1904

3 Weygandt $W$. Über die Mischzustände des manisch-depressiven Irreseins. München: JF Lehmann; 1899

4 Schneider K. Klinische Psychopathologie. Stuttgart: Thieme 1931-2007

5 Himmelhoch JM, Mulla D, Neil JF et al. Incidence and significance of mixed affective states in a bipolar population. Arch Gen Psychiatry 1976; 33: $1062-1066$

6 GoldbergJF, Perlis RH, Bowden CL et al. Manic symptoms during depressive episodes in 1380 patients with bipolar disorder: findings from the STEP-BD. Am J Psychiatry 2009; 166: 173-181

7 Suppes T, Eudicone J, McQuade R et al. Efficacy and safety of aripiprazole in subpopulations with acute manic or mixed episodes of bipolar I disorder. J Affect Disord 2008; 107: 145 - 154

8 Swann AC, Lafer B, Perugi G et al. Bipolar mixed states: an international society for bipolar disorders task force report of symptom structure, course of illness, and diagnosis. Am J Psychiatry 2013; 170: 31 - 42

9 Akiskal HS, Bourgeois ML, Angst J et al. Re-evaluating the prevalence of and diagnostic composition within the broad clinical spectrum of bipolar disorders. J Affect Disord 2000; 59 (Suppl 1): S5-S30

10 Cassidy F, Yatham LN, Berk $M$ et al. Pure and mixed manic subtypes: a review of diagnostic classification and validation. Bipolar Disord 2008; 10: $131-143$
11 Vieta E, Morralla C. Prevalence of mixed mania using 3 definitions. J Affect Disord 2010; 125: $61-73$

12 Balazs J, Benazzi F, Rihmer Z et al. The close link between suicide attempts and mixed (bipolar) depression: implications for suicide prevention. J Affect Disord 2006; 91: 133-138

13 Baldessarini RJ, Salvatore P, Khalsa HM et al. Dissimilar morbidity following initial mania versus mixed-states in type-I bipolar disorder. J Affect Disord 2010; 126: 299-302

14 Cassidy F, Ahearn E, Carroll BJ. A prospective study of inter-episode consistency of manic and mixed subtypes of bipolar disorder. J Affect Disord 2001; 67: 181 - 185

15 Sato T, Bottlender R, Sievers $M$ et al. Evaluating the inter-episode stability of depressive mixed states. J Affect Disord 2004; 81: 103-113

16 Benazzi F. Bipolar disorder-focus on bipolar II disorder and mixed depression. Lancet 2007; 369: 935 -945

17 Pacchiarotti I, Nivoli AM, Mazzarini L et al. The symptom structure of bipolar acute episodes: in search for the mixing link. J Affect Disord 2013; 149: $56-66$

18 Nusslock R, Frank E. Subthreshold bipolarity: diagnostic issues and challenges. Bipolar Disord 2011; 13: 587-603

19 Bschor T, Angst J, Azorin JM et al. Are bipolar disorders underdiagnosed in patients with depressive episodes? Results of the multicenter BRIDGE screening study in Germany. J Affect Disord 2012; 142: 45 - 52

20 Perugi G, Medda P, Reis J et al. Clinical subtypes of severe bipolar mixed states. J Affect Disord 2013; 151: 1076-1082

21 Malhi GS, Lampe L, Coulston CM et al. Mixed state discrimination: a DSM problem that wont go away? J Affect Disord 2014; 158: 8-10

22 Koukopoulos A, Sani G. DSM-5 criteria for depression with mixed features: a farewell to mixed depression. Acta Psychiatr Scand 2014; 129: $4-16$

23 Perugi G, Quaranta G, Dell'Osso L. The significance of mixed States in depression and mania. Curr Psychiatry Rep 2014; 16: 486

24 Pacchiarotti I, Mazzarini L, Kotzalidis GD et al. Mania and depression. Mixed, not stirred. J Affect Disord 2011; 133: 105-113

25 Goldberg JF, Perlis RH, Ghaemi SN et al. Adjunctive antidepressant use and symptomatic recovery among bipolar depressed patients with concomitant manic symptoms: findings from the STEP-BD. Am J Psychiatry 2007; $164: 1348-1355$

26 Köhler S, Gaus S, Bschor T. The challenge of treatment in bipolar depression: evidence from clinical guidelines, treatment recommendations and complex treatment situations. Pharmacopsychiatry 2014; 47: $53-59$

27 Shim IH, Woo YS, Jun TY et al. A reevaluation of the possibility and characteristics in bipolar mania with mixed features: a retrospective chart review. Psychiatry Res 2014; 215: 335-340

28 Gonzalez-Pinto A, Aldama A, Mosquera $F$ et al. Epidemiology, diagnosis and management of mixed mania. CNS Drugs 2007; 21: 611-626

29 Cassidy F, Murry E, Forest $K$ et al. Signs and symptoms of mania in pure and mixed episodes. J Affect Disord 1998; 50: 187-201

30 Swann AC, Secunda SK, Katz MM et al. Specificity of mixed affective states: clinical comparison of dysphoric mania and agitated depression. J Affect Disord 1993; 28: 81 - 89

31 Angst J, Gamma A, Bowden CL et al. Evidence-based definitions of bipolar-I and bipolar-II disorders among 5635 patients with major depressive episodes in the Bridge Study: validity and comorbidity. Eur Arch Psychiatry Clin Neurosci 2013; 263: 663-673

32 Azorin JM, Baraille L, Gerard S et al. Mixed states with predominant manic or depressive symptoms: baseline characteristics and 24-month outcomes of the EMBLEM cohort. J Affect Disord 2013; 146: 369-377

33 Muralidharan K, Ali M, Silveira LE et al. Efficacy of second generation antipsychotics in treating acute mixed episodes in bipolar disorder: a meta-analysis of placebo-controlled trials. J Affect Disord 2013; 150: $408-414$

34 Angst J, Cui L, Swendsen J et al. Major depressive disorder with subthreshold bipolarity in the National Comorbidity Survey Replication. Am J Psychiatry 2010; 167: 1194-1201

35 Swann AC, Bowden CL, Morris D et al. Depression during mania. Treatment response to lithium or divalproex. Arch Gen Psychiatry 1997; 54: $37-42$

36 Bowden CL, Collins MA, McElroy SL et al. Relationship of mania symptomatology to maintenance treatment response with divalproex, lithium, or placebo. Neuropsychopharmacology 2005; 30: 1932 - 1939

37 Bowden CL, Swann AC, Calabrese JR et al. A randomized, placebo-controlled, multicenter study of divalproex sodium extended release in the treatment of acute mania. J Clin Psychiatry 2006; 67: 1501 - 1510 
38 Ghaemi SN, Gilmer WS, Goldberg JF et al. Divalproex in the treatment of acute bipolar depression: a preliminary double-blind, randomized, placebo-controlled pilot study. J Clin Psychiatry 2007; 68: 1840 - 1844

39 Weisler RH, Kalali AH, Ketter TA et al. A multicenter, randomized, double-blind, placebo-controlled trial of extended-release carbamazepine capsules as monotherapy for bipolar disorder patients with manic or mixed episodes. J Clin Psychiatry 2004; 65: 478-484

40 Weisler RH, Keck PE Jr, Swann AC et al. Extended-release carbamazepine capsules as monotherapy for acute mania in bipolar disorder: a multicenter, randomized, double-blind, placebo-controlled trial. J Clin Psychiatry 2005; 66: $323-330$

41 Calabrese JR, Bowden CL, McElroy SL et al. Spectrum of activity of lamotrigine in treatment-refractory bipolar disorder. Am J Psychiatry 1999; 156: 1019-1023

42 Carlson BX, Ketter TA, Sun W et al. Aripiprazole in combination with lamotrigine for the long-term treatment of patients with bipolar I disorder (manic or mixed): a randomized, multicenter, double-blind study (CN138-392). Bipolar Disord 2012; 14: 41 -53

43 Kessing $L V$, Hellmund G, Andersen PK. An observational nationwide register based cohort study on lamotrigine versus lithium in bipolar disorder. J Psychopharmacol 2012; 26: 644-652

44 Amann B, Born C, Crespo JM et al. Lamotrigine: when and where does it act in affective disorders? A systematic review. J Psychopharmacol 2011; 25: $1289-1294$

45 Sachs G, Sanchez R, Marcus R et al. Aripiprazole in the treatment of acute manic or mixed episodes in patients with bipolar I disorder: a 3-week placebo-controlled study. J Psychopharmacol 2006; 20: 536-546

46 Keck PE Jr, Marcus R, Tourkodimitris S et al. A placebo-controlled, double-blind study of the efficacy and safety of aripiprazole in patients with acute bipolar mania. Am J Psychiatry 2003; 160: 1651 - 1658

47 McIntyre RS, Cohen M, Zhao J et al. A 3-week, randomized, placebocontrolled trial of asenapine in the treatment of acute mania in bipolar mania and mixed states. Bipolar Disord 2009; 11: 673-686

48 Azorin JM, Sapin C, Weiller E. Effect of asenapine on manic and depressive symptoms in bipolar I patients with mixed episodes: results from post hoc analyses. J Affect Disord 2013; 145: $62-69$

49 Tohen M, Jacobs TG, Grundy SL et al. Efficacy of olanzapine in acute bipolar mania: a double-blind, placebo-controlled study. The Olanzapine HGGW Study Group. Arch Gen Psychiatry 2000; 57: 841 -849

50 Tohen M, McIntyre RS, Kanba S et al. Efficacy of olanzapine in the treatment of bipolar mania with mixed features defined by DSM-5. J Affect Disord 2014; 168: 136-141

51 Baker RW, Tohen M, Fawcett J et al. Acute dysphoric mania: treatment response to olanzapine versus placebo. J Clin Psychopharmacol 2003, 23: $132-137$

52 Tohen M, Kanba S, McIntyre RS et al. Efficacy of olanzapine monotherapy in the treatment of bipolar depression with mixed features. J Affect Disord 2014; 164: 57-62

53 Tohen M, Sutton VK, Calabrese JR et al. Maintenance of response following stabilization of mixed index episodes with olanzapine monotherapy in a randomized, double-blind, placebo-controlled study of bipolar 1 disorder. J Affect Disord 2009; 116: 43-50
54 Vieta E, Nuamah IF, Lim P et al. A randomized, placebo- and active-controlled study of paliperidone extended release for the treatment of acute manic and mixed episodes of bipolar I disorder. Bipolar Disord 2010; $12: 230-243$

55 Berwaerts J, Melkote R, Nuamah I et al. A randomized, placebo- and active-controlled study of paliperidone extended-release as maintenance treatment in patients with bipolar I disorder after an acute manic or mixed episode. J Affect Disord 2012; 138: 247-258

56 Suppes T, Ketter TA, Gwizdowski IS et al. First controlled treatment trial of bipolar II hypomania with mixed symptoms: quetiapine versus placebo. J Affect Disord 2013; 150: 37 -43

57 Khanna S, Vieta E, Lyons B et al. Risperidone in the treatment of acute mania: double-blind, placebo-controlled study. Br J Psychiatry 2005; 187: $229-234$

58 Singh V, Bowden CL, Mintz J. Relative effectiveness of adjunctive risperidone on manic and depressive symptoms in mixed mania. Int Clin Psychopharmacol 2013; 28: 91 -95

59 Keck PE Jr, Versiani M, Potkin S et al. Ziprasidone in the treatment of acute bipolar mania: a three-week, placebo-controlled, double-blind, randomized trial. Am J Psychiatry 2003; 160: 741 - 748

60 Potkin SG, Keck PE Jr, Segal S et al. Ziprasidone in acute bipolar mania: a 21-day randomized, double-blind, placebo-controlled replication trial. J Clin Psychopharmacol 2005; 25: 301 - 310

61 Stahl S, Lombardo I, Loebel A et al. Efficacy of ziprasidone in dysphoric mania: pooled analysis of two double-blind studies. J Affect Disord 2010; $122: 39-45$

62 Zarate CA Jr, Tohen M. Double-blind comparison of the continued use of antipsychotic treatment versus its discontinuation in remitted manic patients. Am J Psychiatry 2004; 161: 169-171

63 Krüger S, Young T, Bräunig P. Pharmacotherapy of manic-depressive mixed States. Psychiatr Prax 2006; 33 (Suppl 1): S32 - S39

64 Tohen M, Chengappa KN, Suppes T et al. Efficacy of olanzapine in combination with valproate or lithium in the treatment of mania in patients partially nonresponsive to valproate or lithium monotherapy. Arch Gen Psychiatry 2002; 59: 62-69

65 Baker RW, Brown E, Akiskal HS et al. Efficacy of olanzapine combined with valproate or lithium in the treatment of dysphoric mania. Br J Psychiatry 2004; 185: $472-478$

66 Houston JP, Tohen M, Degenhardt EK et al. Olanzapine-divalproex combination versus divalproex monotherapy in the treatment of bipolar mixed episodes: a double-blind, placebo-controlled study. J Clin Psychiatry 2009; 70: $1540-1547$

67 Vieta E, Suppes T, Ekholm B et al. Long-term efficacy of quetiapine in combination with lithium or divalproex on mixed symptoms in bipolar I disorder. J Affect Disord 2012; 142: 36- 44

68 Vieta E, T'Joen C, McQuade RD et al. Efficacy of adjunctive aripiprazole to either valproate or lithium in bipolar mania patients partially nonresponsive to valproate/lithium monotherapy: a placebo-controlled study. Am J Psychiatry 2008; 165: 1316 - 1325

69 Yatham LN, Fountoulakis KN, Rahman Z et al. Efficacy of aripiprazole versus placebo as adjuncts to lithium or valproate in relapse prevention of manic or mixed episodes in bipolar I patients stratified by index manic or mixed episode. J Affect Disord 2013; 147: 365-372 